\title{
Accelerated cine DENSE MRI using compressed sensing and parallel imaging
}

\author{
Xiao Chen ${ }^{1 *}$, Yang Yang ${ }^{1}$, Michael Salerno ${ }^{2,3}$, Craig H Meyer ${ }^{1}$, Frederick H Epstein ${ }^{1,2}$ \\ From 17th Annual SCMR Scientific Sessions \\ New Orleans, LA, USA. 16-19 January 2014
}

\section{Background}

Cine DENSE (Displacement Encoding with Stimulated Echoes) provides accurate and high-resolution displacement and strain imaging of the heart; however, image acquisition times are relatively long and, due to properties inherent to stimulated echoes, signal-to-noise ratio (SNR) is relatively low. Accelerated cine DENSE could substantially shorten scan times and/or provide improved spatial and/or temporal resolution; however, the low SNR, requirement to preserve phase information, and cardiac motion present technical challenges. We aimed to develop acceleration methods that overcome these challenges.

\begin{abstract}
Methods
A variable-density spiral cine DENSE sequence was implemented on a $1.5 \mathrm{~T}$ scanner (Avanto, Siemens) where the center of $\mathrm{k}$-space was fully sampled and the outer portion of k-space was undersampled. Spiral interleaves were distributed uniformly within each cardiac phase and rotated by the golden angle through different cardiac phases. Cine DENSE datasets were collected from 5 healthy volunteers (age 25-28) using a 5-channel RF coil. To make comparisons, fully-sampled cine DENSE datasets with 6 to 8 spiral interleaves per image were acquired within one breathhold (20 to 26 heartbeats), and these datasets were retrospectively rate- 2 undersampled. Also, prospectively rate- 2 undersampled data with 4 spiral interleaves per image were acquired within a shorter breathhold (14 heartbeats). Images had spatial resolution of 1.8-2.2 $\times 1.8-2.2 \times 8 \mathrm{~mm}$ and temporal resolution of $19.6 \mathrm{~ms}$. All undersampled data were reconstructed using a recently-developed compressed sensing (CS) method called Block LOw-rank Sparsity
\end{abstract}

with Motion-guidance (BLOSM), combined with SENSE parallel imaging. The BLOSM-SENSE algorithm exploits matrix low-rank sparsity within motion-tracked regions of SENSE-combined images. Complex-valued images were reconstructed to preserve the phase information used for displacement encoding.

\section{Results}

In Figure 1, example end-systole magnitude- (A-C) and phase-reconstructed (D-F) fully-sampled (A,D), retrospectively-undersampled (B,E), and prospectively-accelerated (C,F) DENSE images and strain maps (G-I) are shown, demonstrating excellent performance of the BLOSMSENSE reconstruction method. Figure 2 shows that quantitation of myocardial displacement by retrospectively rate- 2 accelerated cine DENSE is accurate compared to fully-sampled data. Using prospectively-accelerated cine DENSE, circumferential strain curves from all 5 volunteers demonstrated typical values and patterns for healthy subjects $(\mathrm{C})$.

\section{Conclusions}

Rate-2 accelerated spiral cine DENSE with the BLOSMSENSE reconstruction method provides high-quality complex images. Retrospective undersampling of fullysampled datasets demonstrated accurate displacement and strain measurements. Prospectively undersampled cine DENSE demonstrated typical myocardial strain measurements, using approximately half the data acquisition time of conventional cine DENSE.

\section{Funding}

R01 EB 001763, R01 HL 115225, AHA Award 12PRE12040059 and Siemens Healthcare. 


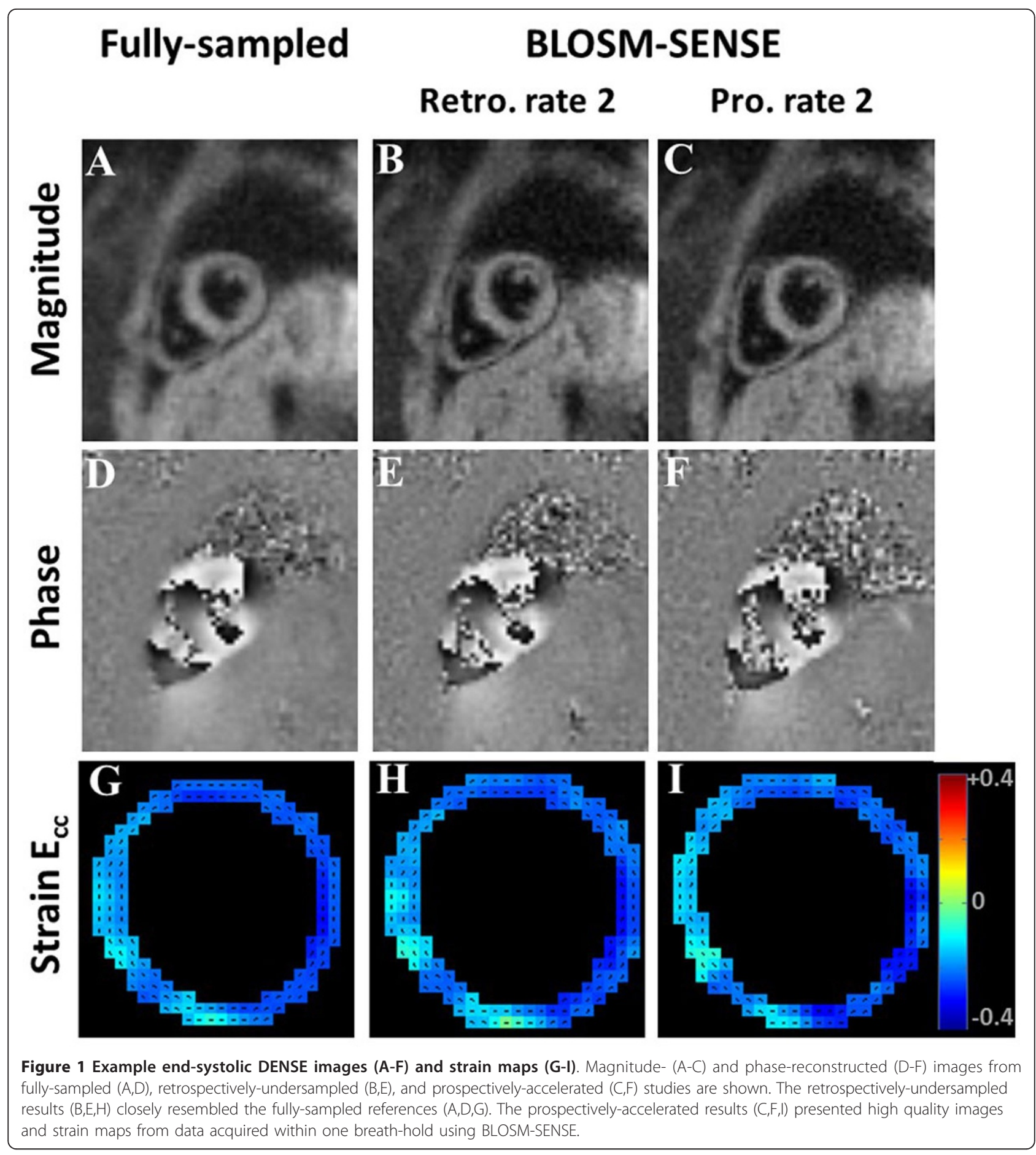




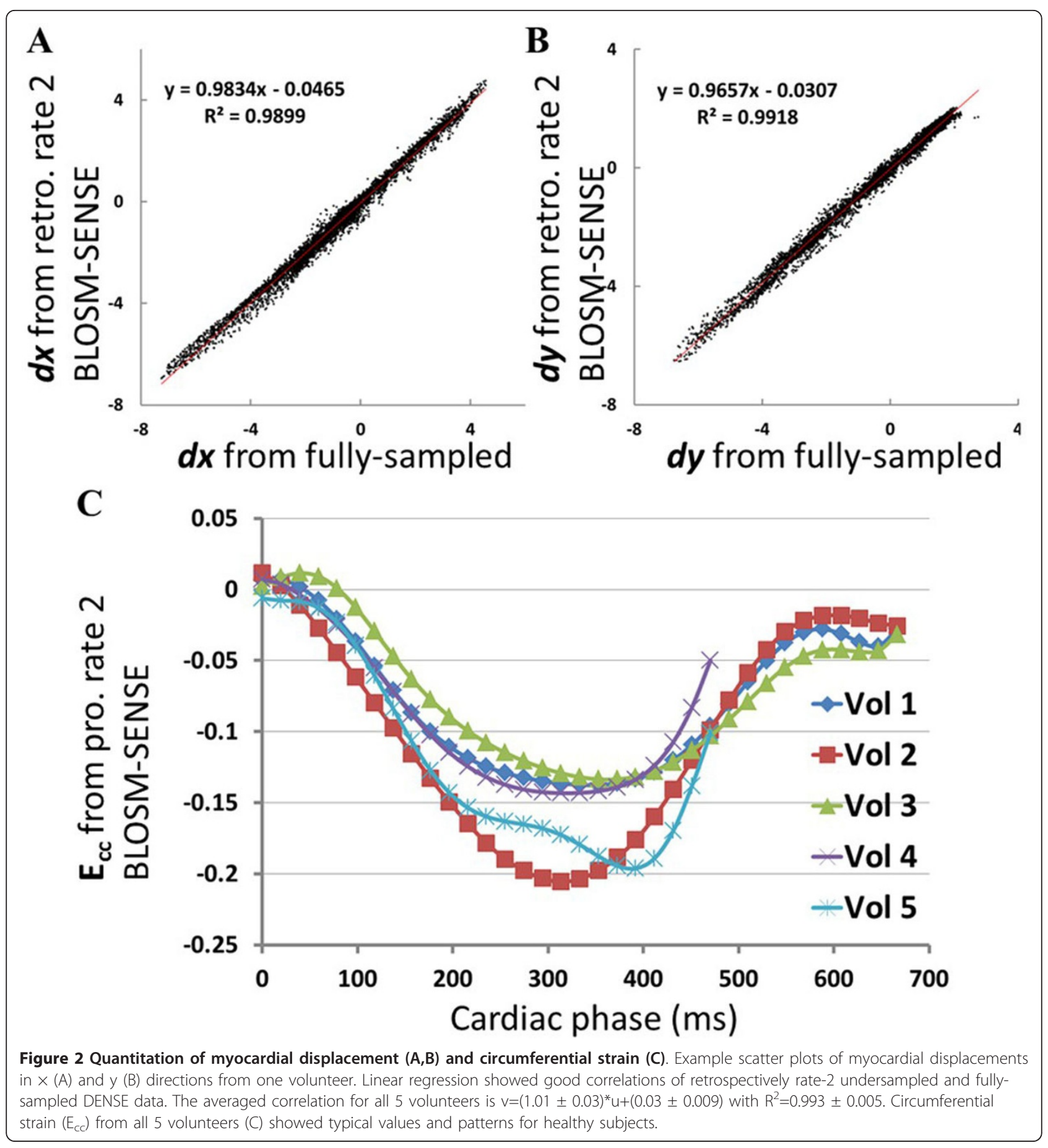

\section{Authors' details}

'Biomedical Engineering, University of Virginia, Charlottesville, Virginia, USA. ${ }^{2}$ Radiology, University of Virginia, Charlottesville, Virginia, USA. ${ }^{3}$ Cardiology, University of Virginia, Charlottesville, Virginia, USA.

Published: 16 January 2014
doi:10.1186/1532-429X-16-S1-W16

Cite this article as: Chen et al:: Accelerated cine DENSE MRI using

compressed sensing and parallel imaging. Journal of Cardiovascular Magnetic Resonance 2014 16(Suppl 1):W16. 